\title{
The relationship between daily physical activity, subjective sleep quality, and mood in sedentary Hungarian adults: A longitudinal within-subjects study
}

\author{
J TAKÁCS ${ }^{1 *}$ and L TÖRÖK ${ }^{2}$ \\ ${ }^{1}$ Faculty of Health Sciences, Semmelweis University, Budapest, Hungary \\ ${ }^{2}$ Department of Psychology and Sport Psychology, University of Physical Education, Budapest, Hungary
}

(Received: October 22, 2019; revised manuscript received: November 17, 2019; accepted: December 2, 2019)

\begin{abstract}
Purpose: This study investigated the day-to-day variability of daily physical activity and its effect on sleep and mood in a longitudinal within-subjects study for 7 days and 6 nights. Materials and methods: Healthy office employees aged 25-35 years with a sedentary lifestyle participated in the study. Seven-day sleep diaries were used to evaluate sleep patterns. Ten-point scales were used to measure the level of happiness and stress. Daily physical activity was measured in steps/day using pedometers. Two hundred forty-five steps/day scores and changes induced in sleep and mood were analysed. Results: There is a relationship between daily physical activity and sleep/mood. An inverted U-shaped relationship may be assumed between sleep duration, sleep quality, feelings after waking up, and the number of steps/day. Increasing the number of steps/day decreases the level of stress and daytime sleepiness and increases sleep efficiency. Sleep efficiency/daytime sleepiness and sleep duration did not show any association. Conclusions: Based on the results, after a physically exhausting day, decreased stress and improved sleep efficiency may be experienced, while sleep duration may decrease, which may reduce the participants' motivation to develop an active lifestyle. For further studies, it would be crucial to use individual exercise intervention programmes to reinforce the positive effects of exercise on sleep and/or mood.
\end{abstract}

Keywords: daily physical activity, sleep quality, stress, pedometer, sedentary

\section{INTRODUCTION}

Several studies have reported that physical activity has a small to moderate sleep-improving effect [1-3], and it has a significant, large, positive effect on subjective sleep quality $[4,5]$. Furthermore, not only can physical activity improve the quality of sleep, but it can also decrease daytime sleepiness [1]; physically active people showed a shorter sleep latency and a longer sleep duration [1-5]. Poor sleep leads to lower levels of physical activity and vice versa [6]; and sleep duration of less than $6 \mathrm{hr}$ results in lower daily physical activity $[5,6]$. In addition, many other factors play a significant role in the relationship between physical activity and sleep, such as motivation, mood, lifestyle, general well-being, and all of these factors can affect sleep itself [7]. Studies have also demonstrated that physical activity has antidepressant and anxiolytic effects [8-10]. Moreover, there is a reciprocal relationship between sleep and mood [11], which, presumably, could also be affected by physical activity. It is also important to mention the fact that body mass index (BMI) is also an important confounding factor in the relationship between physical activity and sleep/mood $[12,13]$, although its specific role has not yet been investigated.

Despite the fact that several studies have reported significant within-person variability for daily physical activity, habitual sleep, and mood [14, 15], studies examining the associations between physical activity and sleep/mood have ignored day-to-day variability, as well as fluctuations in both physical activity and sleep/mood. It is assumed that this variability significantly affects interrelationships between physical activity and sleep/mood [16]; people report a more restful night-time sleep after a day they were more physically active than usual, and vice versa.

To the best of our knowledge, research by Kishida and Elavsky [16] is the only study examining the relationship between daily physical activity and mental health in a longitudinal, within-subjects design. The results of this study indicate that there is a positive relationship between daily physical activity and sleep. On days when the participants' activity counts increased, measured by an accelerometer, longer night-time sleep duration and increased sleep efficiency were found among midlife women.

In previous studies [17, 18], it has been common practice to measure daily physical activity with accelerometers and pedometers. Previous research using pedometers defined the range of inactivity - activity in steps/day, and also demonstrated the reliability of pedometers $[19,20]$. Based on these studies [17, 19-21], fewer than 5,000 steps/day is considered sedentary, 5,000-7,499 steps/day is low active, 7,500-9,999

* Corresponding author: Johanna Takács; Faculty of Health Sciences, Semmelweis University, Vas utca 17, Budapest H-1088, Hungary; E-mails: takacs.johanna@se-etk.hu; spss.stat@gmail.com

This is an open-access article distributed under the terms of the Creative Commons Attribution 4.0 International License, which permits unrestricted use, distribution, and reproduction in any medium, provided the original author and source are credited, a link to the CC License is provided, and changes - if any - are indicated. (SID_1) 
steps/day is somewhat active, 10,000-12,499 steps/day is active, and more than 12,500 steps/day is highly active $[21,22]$. Although pedometers do not provide any information on the intensity of physical activity, they are easy to use, and their reliability has been proven $[19,20]$.

The main purpose of this study is to examine the relationship between daily physical activity and sleep, as well as mood in sedentary adults.

We assessed the effects of the day-to-day variability of daily physical activity objectively measured by a pedometer on subjectively measured sleep and mood through a longitudinal design for 7 days and 6 nights consecutively.

\section{MATERIALS AND METHODS}

\section{Participants}

Participants of this study were aged between 25 and 35 ( $N=35,17$ males and 18 females). The participants were employees of a British bank, working in the Hungarian branch office. They were physically healthy office employees with normal weight $(\mathrm{BMI}=18.5-24.9)$, with a sedentary and inactive lifestyle, and they all worked $8 \mathrm{hr}$ a day, and earned the same salary.

\section{Study measurement}

Assessments prior to the 7-day procedure. Depression was assessed with the 13-item short form of the Beck Depression Inventory (BDI) [23, 24]. Daytime sleepiness was measured using the Epworth Sleepiness Scale (ESS) [25]. Anxiety was assessed with the Spielberger State-Trait Anxiety Inventory (STAI-T/S) [26, 27]. The Hungarian version of the Pittsburgh Sleep Quality Index (PSQI-HUN) was used to obtain subjective reports of sleep [28, 29]. BMI was calculated from weight and height data (BMI = weight in $\mathrm{kg} /$ height in $\mathrm{m}^{2}$ ).

Assessments during the 7-day procedure. Standard 7-day sleep diaries were used both as a source of sleep data and for identifying additional confounding variables. Ten-point Visual Analogue Scales were used to evaluate the level of happiness and stress at the end of each day. Daily physical activity was measured with steps/day by a pedometer (OMRON HJ-005E, OMRON Healthcare Europe B.V., Hungimpex, Ltd., Budapest, Hungary).

\section{Study procedure}

Office employees were recruited through handouts and flyers at their workplace, and the heads of the bank departments also invited employees to participate in this study via e-mail. Following that, the participants received detailed verbal and written information about the purpose and procedure of the study, and about the voluntary basis of their participation. As a next step, they completed the "Assessments before the 7-day procedure", and their weight and height were recorded. In sum, 50 office employees signed up for the study.

Participants with moderate and severe depressive and/or anxiety symptoms were excluded. Furthermore, participants undergoing medical or psychiatric treatments and/or with a
BMI under 18.5 or over 24.9 (underweight or overweight) were also excluded from the 7-day procedure.

In sum, based on the inclusion and exclusion criteria, this study sample was relatively homogenous: healthy office employees with a normal BMI, having a sedentary, inactive lifestyle, with a healthy mood, who performed $8 \mathrm{hr}$ of work daily, with the same salary, aged between 25 and 35 years, both males and females. The homogenous sample ensured the exclusion or control of any confounding variables.

During the 7-day procedure, the subjects measured their steps/day with a pedometer, kept a sleep diary for 7 days, and rated their level of happiness and stress at the end of each day on a 10-point Visual Analogue Scale $(1=$ not at all, $10=$ very)

The 7-day sleep diary consisted of a "Complete in Morning" and a "Complete at the End of Day" sections. "Complete in Morning" included the following variables: bedtime last night, time of awakening, sleep duration (in hr), the number and duration (in min) of awakenings during the night, how long it took to fall back to sleep (in min), use of medication, feelings after waking up (10-point scale: $1=$ fatigued, $10=$ refreshed $)$, and sleep quality $(10$-point scale: $1=$ very poor, $10=$ excellent $)$. "Complete at the End of Day" included the following variables: number of caffeinated/ alcoholic drinks during the day (cups/dl), time of the last caffeinated/alcoholic drink consumption, time and duration of naptimes, time and duration of exercise (if any, in min), and sleepiness during the day (10-point scale, $1=$ struggle to stay awake during the day, $10=$ wide awake during the day).

To register steps/day, a pedometer was used. Before starting the 7-day procedure, participants were familiarised with the pedometer and the study procedure. They were given the following instructions: "The aim of our study is NOT to increase your daily activity, but simply to register your normal daily activity, your steps/day for a week. The pedometer will record every step when moving around for more than ten minutes."

After the 7-day procedure, participants were personally interviewed on whether there had been any important positive or negative events in their lives or any acute illnesses during the 7-day procedure. Participants received both oral and written information about the study, and they signed an informed consent form.

\section{Data analyses}

For describing data, distribution of relative frequencies and descriptive analyses with means and standard deviations were used. For examining statistical differences between males and females, independent samples $t$-tests were applied; for interpreting the results in practice, bias-corrected Hedges' $g$ was used to measure the effect size with confidence interval calculated. For examining the statistical differences between three samples (based on steps/day scores), one-way analysis of variance was performed with the calculation of omega squared effect size (with confidence interval). For measuring associations between variables, Spearman's rank-correlation coefficients were calculated. All statistical procedures were performed using IBM SPSS Statistics for Windows, version 23.0 (IBM Corp., Released 2015, Armonk, NY, USA). 


\section{RESULTS}

\section{Participants' characteristics}

At the baseline of the 7-day procedure. The sample included 35 participants (18 females and 17 males), who were office employees with a sedentary, inactive lifestyle. The mean age of participants was 34.6 years $(S D=2.9)$. Based on the PSQI-HUN, the sleep quality of participants indicated poor sleep, and participants showed small, non-disruptive daytime sleepiness based on ESS. All participants were in a normal mood state (based on the BDI and the STAI-T/S minimal depression and/or low/moderate state/trait anxiety) and by normal weight (BMI range: 18.60-28.98; Table 1). There were no statistically significant differences in any variables between males and females.

Results of the 7-day procedure. Based on the results of the sleep diaries, participants did not perform any further exercise on top of their usual daily physical activity. None of the participants were treated by medication during the 7-day procedure. The amount of caffeinated/alcoholic drinks consumed was unchanged over the course of the 7 days (five people had caffeinated drinks with a mean of 1.31 cups/day, and these drinks were consumed before 5 p.m.).

\section{Steps/day and definition of the steps/day scores}

The mean of steps/week was 8,526.7 steps/day $(S D=$ 7780.9). There was no statistically significant difference between males and females in steps/day over a week $[t(33)=-0.432, p=.667)]$. When studying the relationship between steps/day and the variables of sleep and mood, 245 steps/day scores were examined (the number of participants was 35 , the procedure was continued for 7 days; therefore, the number of cases $35 \times 7=245$ steps/day scores). The next step was to categorise the number of steps/day based on the individual mean of steps/week (the number of daily steps divided by the number of weekly steps for each participant). Steps/day scores lower than $80 \%$ of the number of weekly steps were categorised as below-the-usual steps/day scores. Steps/day scores between $80 \%$ and $120 \%$ of the number of weekly steps were categorised as usual steps/day scores, and those over $120 \%$ of the number of weekly steps were categorised as above-the-usual steps/day scores. For example, John had a mean of 7,818 steps/day over a week. On the first day, he took 10,654 steps; this was $136 \%$ of the mean of his weekly steps $(7,818)$, resulting in an above-the-usual steps/day score. On the second day, he took 5,596 steps, which was $72 \%$ of the mean of his weekly steps, giving him a below-the-usual steps/day score, and so on. In sum, $47.8 \%$ of the recorded steps for a week were usual steps/day scores $(n=117), 31.4 \%$ were below-theusual steps/day scores $(n=77)$, and $20.8 \%$ were above-theusual steps/day scores $(n=51)$.

The effects of steps/day scores on a certain day were measured in terms of the induced changes in the measured variables on the same day. These variables were measured at the end of day (daytime sleepiness, levels of stress, and happiness), or they were evaluated in the morning (sleep efficiency, sleep duration, feelings after waking up, and sleep quality).

\section{Results of sleep variables}

Based on the 7-day sleep diaries, the following sleep variables were examined: sleep duration (bedtime - time of awakening), sleep efficiency (time in bed/sleep duration in $\%)$, feelings after waking up (10-point scale, $1=$ fatigued, $10=$ refreshed $)$, sleep quality $(10$-point scale, $1=$ very poor , $10=$ excellent $)$, and daytime sleepiness (10-point scale, $1=$ struggle to stay awake during the day, 10= wide awake during the day).

Sleep duration. The mean of the sleep duration was $7.0 \mathrm{hr}$ a day $(S D=1.4)$. There were significant differences between the below-the-usual, usual, and above-the-usual steps/day scores in sleep duration $[F(2,242)=11.948$, $\left.p<.001, \omega^{2}=0.34(0.11,0.53)\right]$. Sleep duration in the below-the-usual steps/day scores $(p<.001)$ and in the above-the-usual steps/day scores $(p=.044)$ was significantly shorter than in the usual steps/day scores. However, there was no significant difference between the below-the-usual and the above-the-usual steps/day scores in sleep duration $(p=.170 ;$ Figure 1). The relationship between steps/day and sleep duration can be described with an inverted U-shaped curve.

Sleep efficiency. The mean of sleep efficiency was $86.3 \%$, which shows excellent efficiency. Simultaneously, sleep efficiency was a heterogeneous variable with a standard deviation of $16.6 \%$, and at times its value was higher than $100 \%$ due to overestimation.

Table 1. Participants' characteristics in the total sample and in the groups of males and females

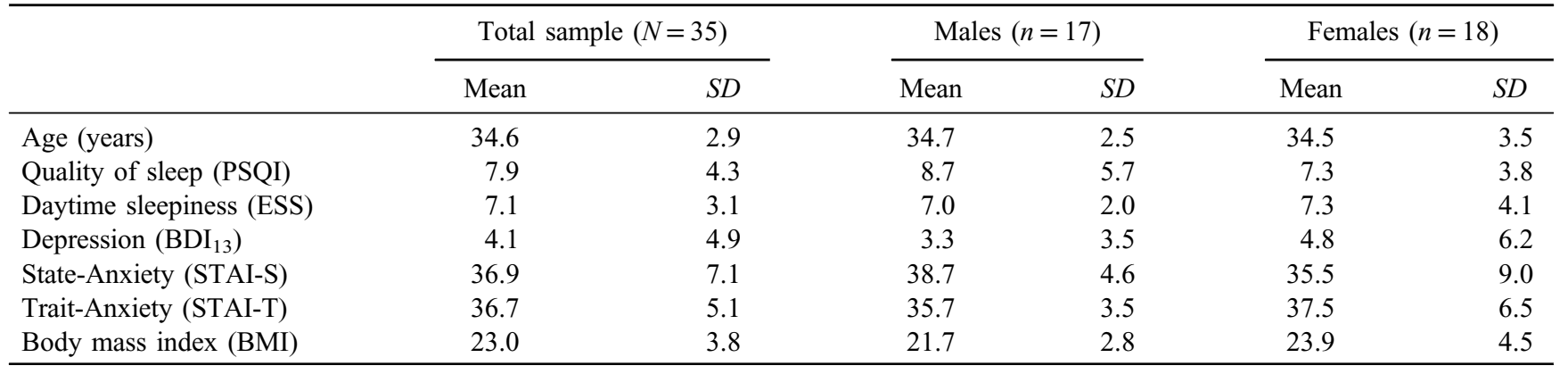

Note. SD: standard deviation; ESS: Epworth Sleepiness Scale; PSQI: Pittsburgh Sleep Quality Index; STAI: State-Trait Anxiety Inventory; BDI: Beck Depression Inventory. 


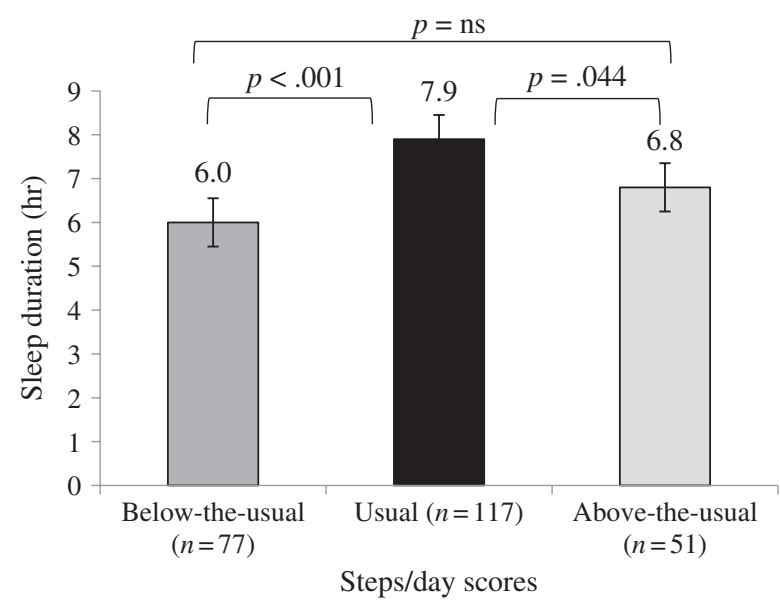

Figure 1. The mean of sleep duration in the below-the-usual, usual, and above-the-usual steps/day scores (ns: non-significant; error bars: standard error)

Based on the results, there was a significant, positive, weak monotonous relationship between sleep efficiency and steps/day $[\rho(243)=0.394, p=.006]$. This means that if a person reported a high number of steps a day, they evaluated their sleep that night as more restful, than after a lower number of steps a day, and vice versa. The mean of sleep efficiency was $70.8 \%(S D=15.9 \%)$ in the below-the-usual steps/day scores, $91.8 \%(S D=12.1 \%)$ in the usual steps/day scores, and $96.8 \%(S D=9.8 \%)$ in the above-the-usual steps/day scores. There was a non-significant association between sleep efficiency and sleep duration $[\rho(243)=.230$, $p=.117]$.

Feelings after waking up - "fatigued-refreshed". The mean of feelings after waking up rated on a 10-point scale was $5.8(S D=2.4)$. About $4.2 \%$ of participants reported "fatigued" (value 1) and 8.3\% reported "refreshed" (value 10). Statistically significant differences were found between steps/day scores in "fatigued-refreshed" $[F(2,242)=5.445$, $\left.p=.012, \omega^{2}=0.12(0.00,0.30)\right]$. The lowest value of "fatigued-refreshed" was found in the below-the-usual steps/day scores and the highest value was found in the usual steps/day scores $(p=.006)$; in the above-the-usual steps/day scores, "fatigued-refreshed" had an intermediate value (Figure 2). The relationship between steps/day and feelings after waking up can be described with an inverted U-shaped curve.

Sleep quality. Participants rated their sleep quality on a 10 -point scale $(M=7.9, S D=2.2)$. About $4.2 \%$ of participants showed very poor sleep quality (value 1) and $22.9 \%$ reported excellent sleep quality (value 10). The steps/day scores did not show any statistically significant differences in sleep quality $\left[F(2,45)=3.224, p=.062, \omega^{2}=0.09(0.00\right.$, $0.26)$ ]. Sleep quality was the lowest in the below-the-usual steps/day scores and the highest in the usual steps/day scores $(p=.038)$. Sleep quality had an intermediate value in the above-the-usual steps/day scores (Figure 3). The relationship between steps/day and sleep quality can be described with an inverted U-shaped curve.

Daytime sleepiness. The mean of daytime sleepiness (rated on a 10-point scale) was $4.4(S D=1.8)$. There was a significant, negative, moderate monotonous relationship

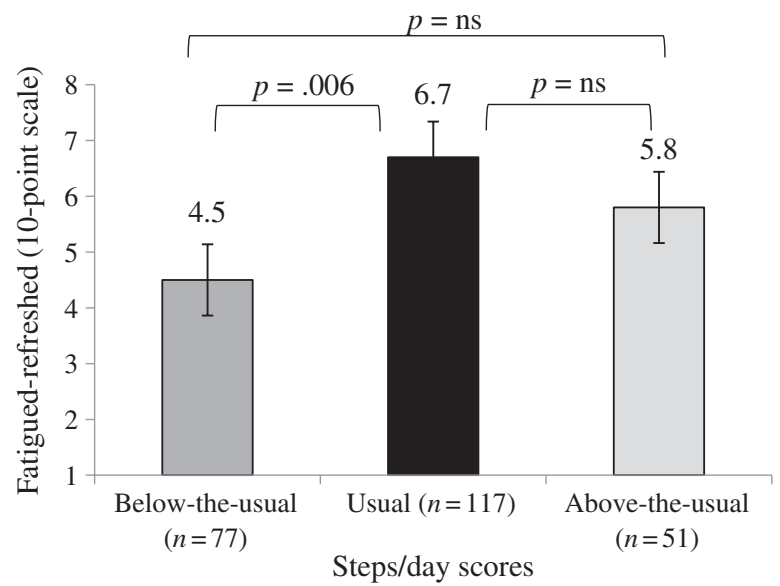

Figure 2. The mean of feelings after waking up in the below-theusual, usual, and above-the-usual steps/day scores

(ns: non-significant; error bar: standard error)

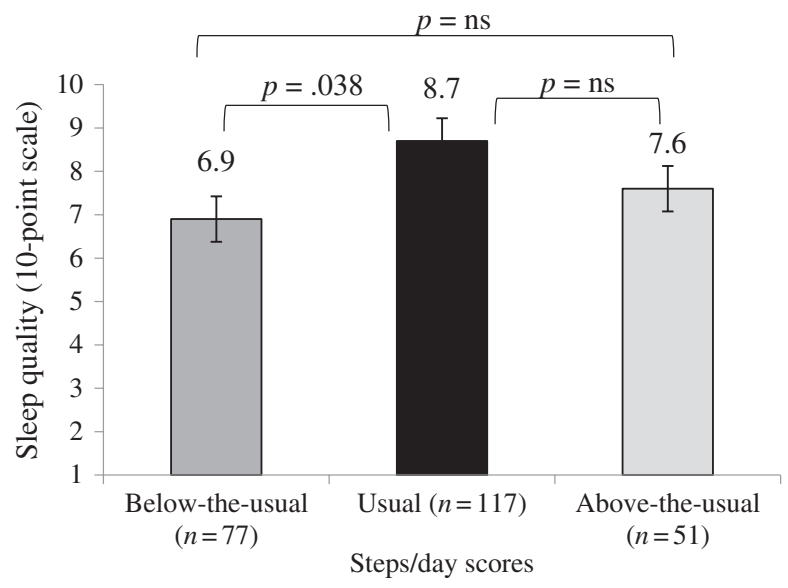

Figure 3. The mean of sleep quality in the below-the-usual, usual, and above-the-usual steps/day scores (ns: non-significant; error bar: standard error)

between daytime sleepiness and steps/day $[\rho(245)=$ $-0.517, p<.001]$. This means that increasing the number of daily steps decreases daytime sleepiness and vice versa. The mean of daytime sleepiness was $5.9(S D=1.4)$ in the above-the-usual steps/day scores, $4.3(S D=1.5)$ in the usual steps/day scores, and $2.4(S D=1.0)$ in the abovethe-usual steps/day scores (Figure 4). There was a nonsignificant association between daytime sleepiness and sleep duration $[\rho(243)=-0.002, p=.989]$.

\section{Results of mood variables}

Participants estimated the levels of both their happiness and stress at the end of every day on a 10-point Visual Analogue Scale $(1=$ not at all, $10=$ very $)$.

Happiness. There was a non-significant correlation between happiness and steps/day $[\rho(243)=-0.194, p=.187]$. The mean of happiness was $6.9(S D=1.3)$ in the below-theusual steps/day scores, $6.7(S D=1.7)$ in the usual steps/day scores, and $7.0(S D=1.6)$ in the above-the-usual steps/day scores. 


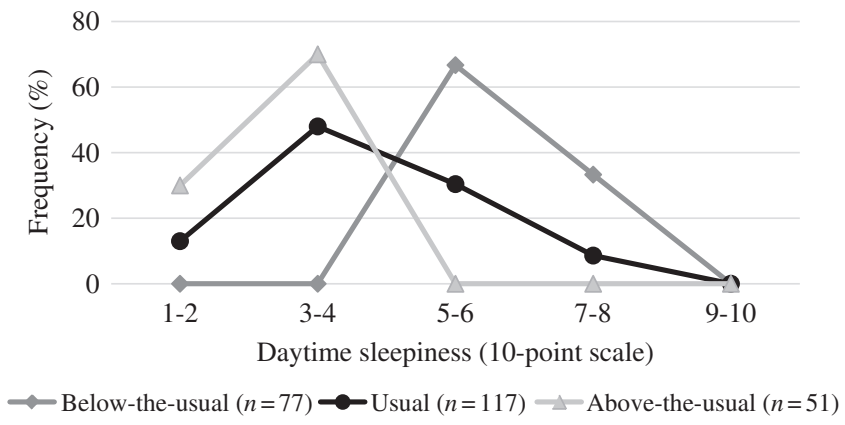

Figure 4. Frequency distribution of daytime sleepiness in the below-the-usual, usual, and above-the-usual steps/day scores

Above-the-usual $(n=51)$ 50

Usual $(n=117)$ 43.4 \begin{tabular}{l|l|l|l}
\hline 21.8 & 17.4 & 8.78 .7
\end{tabular}

\begin{tabular}{|c|c|c|c|c|c|}
\hline \multirow[t]{3}{*}{ Below-the-usual $(n=77)$} & ) 13.4 & 13.4 & 20 & \multicolumn{2}{|l|}{53.2} \\
\hline & $0 \%$ & $20 \%$ & $40 \%$ & $60 \%$ & $100 \%$ \\
\hline & \multicolumn{5}{|c|}{ Stress (10-point scale) } \\
\hline
\end{tabular}

$\square$ 1-2 $\square 3-4 \quad \square 5-6 \quad \square 7-8 \quad \square 9-10$

Figure 5. Frequencies of the level of stress in the below-the-usual, usual, and above-the-usual steps/day scores (values 1 and 2 mean: "I did not feel any stress")

Stress. There was a significant, negative, strong correlation between stress and steps/day $[\rho(243)=-0.717$, $p<.001]$; consequently, increasing the number of steps/day decreases the level of stress, and vice versa. In the belowthe-usual steps/day scores, the mean of stress was 5.7 $(S D=2.2) ; 13.4 \%$ of these scores fell into the category of "I did not feel any stress" (values 1 and 2). In the usual steps/day scores, the mean of stress was $3.9(S D=2.4)$, and $43.4 \%$ of the scores showed values 1 or 2 . However, in the above-the-usual steps/day scores, the mean of stress was 2.5 $(S D=1.1)$. One half of cases had the values of 1 or 2 , and for the other half of cases, the level of stress was marked with values 3 or 4 (Figure 5).

\section{DISCUSSION}

This study examined the fluctuation of daily physical activity recorded by pedometers in sedentary adults as well as the changes induced in sleep and mood. Based on the results, an inverted U-shaped relationship may be assumed between sleep duration, sleep quality, feelings after waking up, and the number of steps/day in the study sample. Inactive subjects, who recorded step numbers less than $80 \%$ or higher than $120 \%$ on a usual day, reported shortened sleep duration and worse-than-usual sleep quality at night and felt more fatigued and sleepier after getting up on the following day. In addition, increasing daily physical activity (more steps/day) increased the participants' efficiency of sleep at night and decreased their daytime sleepiness on the following day. It is important to note that there was no association found between sleep efficiency/daytime sleepiness and sleep duration. Moreover, increasing daily physical activity significantly decreased the participants' level of stress.

Kishida and Elavsky [16] were the first to examine the relationship between daily physical activity and health in midlife women. Based on their results, there is a positive relationship between daily physical activity and sleep duration. Our findings are partly consistent with these results; however, a direct comparison is impossible to make due to the differences both in sample and study design [16].

There are a few notable limitations to this study. First, our sample was relatively small and homogenous. The required sample size was calculated using the software $\mathrm{G}^{*}$ Power 3.1 [30], considering an effect size of $0.5, \alpha$ of 0.05 , and $1-\beta$ of 0.8 . A homogenous sample was important in the study design to handle the potential mediators and moderators in the relationship between physical activity and sleep/mood, such as BMI and moderate/severe symptoms of depression and/or anxiety. Evidently, there is an interrelationship between insomnia disorder/symptoms and mood disorders/symptoms [31-33], and BMI is also often considered to be interrelated with sleep and mood $[12,13]$ as well as physical activity [16]. Second, sleep was measured subjectively with sleep diaries to evaluate changes in sleep patterns for 7 days. Nevertheless, a sleep diary is a standard and reliable tool for monitoring and assessing sleep subjectively, and it is suitable for studying within-person day-today changes of subjective sleep [34].

In sum, even considering these limitations, this study demonstrated that there is a significant relationship between daily physical activity and sleep/mood. It can be assumed that there is an inverted U-shaped relationship between daily physical activity and sleep duration, sleep quality, and feelings after waking up. Furthermore, there is a positive relationship between daily physical activity and sleep efficiency as well as daytime sleepiness, and there is a negative relationship between daily physical activity and the level of stress. These associations can be detected even if variables are measured subjectively.

\section{CONCLUSIONS}

Studying the relationship between daily physical activity and sleep/mood is important for at least two reasons. First, defining the "dose-response" of health-enhancing physical activity is an important focus area all around the world. Second, the most commonly performed health-enhancing physical activity available to the masses is walking $[35,36]$. Therefore, not only is it important to know how many steps/day are required to maintain adequate physical activity, but it is also crucial to examine what it may lead to if the number of daily steps is suddenly (intentionally/ spontaneously) increased. Although the currently used recommendations of physical activity emphasise active lifestyle [37], they do not provide any specific instructions on how to increase the amount of physical activity gradually and how to achieve and maintain an active lifestyle (dose-response). 
This study also underpins the fact that if people want to achieve their goals (active lifestyle) too fast, they may overburden themselves. In that case, the positive effects of physical activity may be manifested in decreased stress levels and improved sleep efficiency; yet, sleep duration may also decrease resulting in increased fatigue the following day. Consequently, these changes may reduce one's motivation to continue regular physical activity/exercise. This is crucial as the dropout rate of exercise intervention programmes is high, potentially as high as $90 \%$ even in the case of an intervention where the goal is "only" to maintain $10,000 \mathrm{steps} /$ day [38].

Consequently, there are many advantages of using a well-planned, individualised exercise intervention programme. With such programmes, an active lifestyle can be gradually developed, while keeping the psychological indicators in their optimal zone. By providing an adequate amount of exercise, the positive effects on sleep and mental/ somatic well-being may also be reinforced.

Authors' contribution: JT summarized the scientific background of the paper, collected the data, and carried out the statistical analysis. LT involved in interpretation of data for the work, critical revision of the manuscript, and finalizing the text.

Ethical approval: All procedures were in accordance with the ethical standards of the institutional and/or national research committee and with the 1964 Helsinki Declaration and its later amendments or comparable ethical standards (TE-KEB/No26/2019).

Conflicts of Interest/Funding: The authors declare no conflict of interest and no financial support was received for this study.

\section{REFERENCES}

1. Loprinzi PD, Cardinal BJ. Association between objectively measured physical activity and sleep, NHANES 2005-2006. Ment Health Phys Act. 2011;4(2):65-9.

2. Kline CE. The bidirectional relationship between exercise and sleep: implications for exercise adherence and sleep improvement. Am J Lifestyle Med. 2014;8(6):375-9.

3. Dolezal BA, Neufeld EV, Boland DM, Martin JL, Cooper CB. Interrelationship between sleep and exercise: a systematic review. Adv Prev Med. 2017;2017:1364387.

4. Yang PY, Ho KH, Chen HC, Chien MY. Exercise training improves sleep quality in middle-aged and older adults with sleep problems: a systematic review. J Physiother. 2012;58(3): 157-63.

5. Kredlow MA, Capozzoli MC, Hearon BA, Calkins AW, Otto MW. The effects of physical activity on sleep: a meta-analytic review. J Behav Med. 2015;38(3):427-49.

6. Holfeld B, Ruthig JC. A longitudinal examination of sleep quality and physical activity in older adults. J Appl Gerontol. 2014;33(7):791-807.
7. Driver HS, Taylor SR. Exercise and sleep. Sleep Med Rev. 2000;4(4):387-402.

8. Conn VS. Depressive symptom outcomes of physical activity interventions: meta-analysis finding. Ann Behav Med. 2010;39(2):128-38.

9. Stanton R, Reaburn P. Exercise and the treatment of depression: a review of the exercise program variables. J Sci Med Sport. 2014;17(2):177-82.

10. Rebar AL, Stanton R, Geard D, Short C, Duncan MJ, Vandelanotte C. A meta-meta-analysis of the effect of physical activity on depression and anxiety in non-clinical adult populations. Health Psychol Rev. 2015;9(3):366-78.

11. Konjarski M, Murray G, Lee VV, Jackson ML. Reciprocal relationships between daily sleep and mood: a systematic review of naturalistic prospective studies. Sleep Med Rev. 2018;42:47-58.

12. Annesi JJ. Relations of mood with body mass index changes in severely obese women enrolled in a supported physical activity treatment. Obes Facts. 2008;1(2):88-92.

13. Ogilvie RP, Patel SR. The epidemiology of sleep and obesity. Sleep Health. 2017;3(5):383-8.

14. Matthews CE, Ainsworth BE, Thompson RW, Bassett DR Jr. Sources of variance in daily physical activity levels as measured by an accelerometer. Med Sci Sports Exerc. 2002;34(8):1376-81.

15. Bei B, Wiley JF, Trinder J, Manber R. Beyond the mean: a systematic review on the correlates of daily intraindividual variability of sleep/wake patterns. Sleep Med Rev. 2016;28: 108-24.

16. Kishida M, Elvasky S. An intensive longitudinal examination of daily physical activity and sleep in midlife women. Sleep Health. 2016;2(1):42-8.

17. Hatano Y. Use of the pedometer for promoting daily walking exercise. ICHPER J. 1993;29:4-8.

18. Freedson PS, Miller K. Objective monitoring of physical activity using motion sensors and heart rate. Res Q Exerc Sport. 2000;71(2):S21-9.

19. Bassett DR Jr, Ainsworth BE, Leggett SR, et al. Accuracy of five electronic pedometers for measuring distance walked. Med Sci Sports Exerc.1996;28(8):1071-7.

20. Tudor-Locke C, Williams JE, Reis JP, Pluto D. Utility of pedometers for assessing physical activity: convergent validity. Sports Med. 2002;32(12):795-808.

21. Tudor-Locke C, Hatano Y, Pangrazi RP, Kang M. Revisiting "how many steps are enough"? Med Sci Sports Exerc. 2008;40(7):537-43.

22. Choi CK, Pak AWP, Choi JCL, Choi ECL. Daily step goal of 10.000 steps: a literature review. Clin Invest Med. 2007;30(3): E146-51.

23. Beck AT, Beck RW. Shortened version of BDI. Post Grad Med. 1972;52:81-5.

24. Rózsa S, Szádóczky E, Füredi J. A Beck Depresszió Kérd ív rövidített változatának jellemz i a hazai mintán [Shortened version of BDI in Hungarian sample]. Psychiatr Hung. 2001;16(4):379-97.

25. Johns MW. A new method for measuring daytime sleepiness: the Epworth Sleepiness Scale. Sleep. 1991;14:540-5.

26. Spielberger CD, Gorsuch RL, Lushene RE. Manual for the State-Trait Anxiety Inventory. Palo Alto, CA: Consulting Psychologist Press; 1970.

27. Sipos K, Sipos M. The development and validation of the Hungarian form of the STAI. In: Spielberger CD, 
DiazGuerrero R, eds. Cross-Cultural Anxiety, 2. Washington/ London: Hemisphere; 1978. p. 51-61.

28. Buysse DJ, Reynolds CF, Monk TH, Berman SR, Kupfer DJ. The Pittsburgh Sleep Quality Index: a new instrument for psychiatric practice and research. Psychiatry Res. 1989;28(2): 193-213.

29. Takács J, Bódizs R, Ujma PP, Horváth K, Rajna P, Harmat L. Reliability and validity of the Hungarian version of the Pittsburgh Sleep Quality Index (PSQI-HUN): comparing psychiatric patients with control subjects. Sleep Breath. 2016;20(3):1045-51.

30. Faul F, Erdfelder E, Lang A-G, Buchner A. A flexible statistical power analysis program for the social, behavioral, and biomedical sciences. BRM. 2007;39(2):175-91.

31. Buysse DJ, Angst J, Gamma A, Ajdacic V, Eich D, Rössler W. Prevalence, course, and comorbidity of insomnia and depression in young adults. Sleep. 2008;31(4):473-80.

32. Mayers AG, Grabau EA, Campbell C, Baldwin DS. Subjective sleep, depression and anxiety: inter-relationships in a non-clinical sample. Hum Psychopharmacol. 2009;24(6): 495-501.
33. Alvaro PK, Roberts RM, Harris JK. A systematic review assessing bidirectionality between sleep disturbances, anxiety, and depression. Sleep. 2013;36(7):1059-68.

34. Carney CE, Buysse DJ, Ancoli-Israel S, et al. The consensus sleep diary: standardizing prospective sleep self-monitoring. Sleep. 2012;35(2):287-302.

35. Lee IM, Buchner DM. The importance of walking to public health. Med Sci Sports Exerc. 2008;40(7):S512-8.

36. Tudor-Locke C, Swift DL, Schuna JM Jr, et al. WalkMore: a randomized controlled trial of pedometer-based interventions differing on intensity messages. BMC Public Health. 2014;14:168.

37. WHO. Global Action Plan on Physical Activity 2018-2030: More Active People for a Healthier World. [Internet]. Geneva: World Health Organization; 2018 [cited 2019 Oct 2]. Available from: http://apps.who.int/iris/bitstream/handle/10665/ 272722/9789241514187eng.pdf?ua=1

38. Iwane M, Arita M, Tomimoto S, et al. Walking 10,000 steps/ day or more reduces blood pressure and sympathetic nerve activity in mild essential hypertension. Hypertens Res. 2000;23(6):573-680. 